\title{
Unbalanced international collaboration affects adversely the usefulness of countries' scientific output as well as their technological and social impact
}

\author{
Sonia R. Zanotto ${ }^{1} \cdot$ Cristina Haeffner $^{2} \cdot$ Jorge A. Guimarães $^{3}$
}

Received: 30 April 2016/Published online: 17 September 2016

(C) The Author(s) 2016. This article is published with open access at Springerlink.com

\begin{abstract}
The unbalanced international scientific collaboration as cause of misleading information on the country's contribution to the scientific world output was analyzed. ESI Data Base (Thomson Reuters' InCites), covering the scientific production of 217 active countries in the period 2010-2014 was used. International collaboration implicates in a high percentage $(33.1 \%$ ) of double-counted world articles, thus impacting qualitative data as citations, impact and impact relative to word. The countries were divided into three groups, according to their individual contribution to the world publications: Group I (24 countries, at least $1 \%$ ) representing $83.9 \%$ of the total double-counted world articles. Group II (40 countries, $0.1-0.99 \%$ each). Group III, 153 countries $(70.5 \%$ ) with $<0.1 \%$ and altogether $1.9 \%$ of the world. Qualitative characteristics of each group were also analyzed: percentage of the country's GNP applied in R\&D, proportion of Scientists and Engineers per million inhabitants and Human Development Index. Average international collaboration were: Group I, $43.0 \%$; Group II, $55.8 \%$ and Group III, 85.2 \%. We concluded that very high and unbalanced international collaboration, as presented by many countries, misrepresent the importance of their scientific production, technological and social outputs. Furthermore, it jeopardizes qualitative outputs of the countries themselves, artificially increasing their scientific impact, affecting all fields and therefore, the whole world. The data confirm that when dealing with the qualitative contribution of countries, it
\end{abstract}

Sonia R. Zanotto and Cristina Haeffner have contributed equally to this work.

Jorge A. Guimarães

jguimaraes14@gmail.com

1 Programa de Pós-Graduação em Educação em Ciências: Química da Vida e Saúde (PPGQVS), Universidade Federal do Rio Grande do Sul - UFRGS and Instituto Brasileiro de Geografia e Estatística (IBGE - RS), Porto Alegre, RS, Brazil

2 Biblioteca do Instituto de Educação e Pesquisa (IEP), Hospital Moinhos de Vento, Porto Alegre, RS, Brazil

3 Hospital de Clínicas de Porto Alegre and Centro de Biotecnologia, Universidade Federal do Rio Grande do Sul - UFRGS, Av. Bento Gonçalves, 9500, C.P. 15005, CEP 91501-970 Porto Alegre, RS, Brazil 
is necessary to take in consideration the level of international cooperation because, as seen here, it can and in fact it does create false impression of the real contribution of countries.

Keywords Scientific production · Impact · International collaboration ·

Double-counting of articles $\cdot$ Bibliometric analysis

\section{Introduction}

Today, most countries, no matter how developed they are, produce scientific articles in several areas and specific fields of knowledge. In fact, as observed by the Australian Academy of Sciences (2010), "Developing countries have increased their participation in science and technology research" and thus "scientists from more developed countries are building personal partnerships with these countries". Presently, a total of 251 specialized fields are listed in the Web of Science (Thomson Reuters' InCites Data Base), and a total of 217 countries are publishing scientific articles. According to the ESI Data Base, the 251 fields are summarized in 22 great areas of knowledge. Although the number of countries publishing articles in each specific field can vary largely, very few among the 22 areas have less than one hundred countries contributing with research and publications. On the other hand, the great majority of scientific articles are published by a small group of countries most occupying the highest level of economic welfare and social development, thus corroborating the notion that "research and innovation contribute in the short and long terms to prosperity and competitiveness, as well as to the resolution of society's greatest challenges in areas like health, energy and security" (Wadworth 2014). In this context it is important to consider the thought of William Press (Press 2013) about the social impact of science in the USA: "what is so special about science (and how much we should spend on it)?"

Scientific international collaboration, as measured by co-authorship and publications has been growing constantly and the percentage of co-authored articles has more than doubled in the last two decades (Wagner et al. 2015). These authors observed that the open possibility of co-authorship has attracted productive scientists to participate in international projects. Nevertheless, they emphasized that "National governments could gain efficiencies and influence by developing policies and strategies designed to maximize network benefits - a model different from those designed for national systems". It has been also emphasized that international co-authored publications receive greater number of citations (Glänzel and Schubert 2001; Persson et al. 2004). This distinctive pattern also occurs in more developed countries like the Europeans ones (Narin et al. 1991). Such considerations are important issues for the analysis presented in this work.

Since we studied the proportion of international collaboration pattern as a whole, our findings could not contradict the notion that "the more basic the field, the greater the proportion of international co-authorships" (Frame and Carpenter 1979; Luukkonen et al. 1992). Also, we did not distinguish between collaboration and co-authorship as emphasized by Katz and Martin (1997). Actually, as mentioned by Subramanyam (1983), "the assessment of collaboration using co-authorship is by no means perfect, it nevertheless has certain advantages: it is invariant and verifiable; given access to the same data-set, other investigators should be able to reproduce the results". Several authors have shown that international collaboration increases visibility of the scientific work (Katz and Martin 
1997) and the resultant publications receive higher consideration (Van Raan 1988) or, in other words, publications resulting from international collaboration produce "more positive effects on the quality of the output when compared to research without collaboration" (Abramo et al. 2009). Other authors made similar observations (Meneghini 2010; Tahamtan et al. 2016; Meneghini et al. 2008; Akre et al. 2011; Leite et al. 2011). Concerning to this, Smith et al. (2014) studying journal placement and citation performance of articles, observed that "the relative success of articles can be holistically assessed, yielding new insights into the scientific impact of individual countries and cross-national collaboration". As a consequence, it can be concluded that international collaboration produces a number of benefits for participating researchers "that ultimately translate into greater scientific visibility, quality and impact". In fact this has been shown by several authors (Van Raan 1988; Martin-Sempere et al. 2002; Barjack and Robinson 2007; Bozeman and Corley 2004) allowing to conclude that "international collaboration produces real and remarkable results in the scientific performance of research groups" (Abramo et al. 2009; Gevers 2014). Actually, the subject of cooperation can be considered an international enterprise (Van Raan 1997). This is especially true when it includes highly productive scientists from the most developed countries. In such cases there is a great chance that articles will be published in a journal with a higher impact factor, thus resulting in significant increase in citations and thus of the impact of the publication (Katz and Martin 1997; Abramo et al. 2009; Smith et al. 2014). This phenomenon has to do with the known effect of authors' affiliation (Akre et al. 2011). On the other hand for the less developed countries international collaboration is based in the concept assumed by funding agencies and policy makers that it is "a good thing" that "should be universally encouraged" (Katz and Martin 1997). Furthermore, it is well known that the number of published articles in a specific field depends on the size of the scientific community of that area or field (Gaffriau and Larsen 2005). This has been shown by Smith et al. (2014), who analyzed eight different disciplines in relation to two categories of indicators: journal placement and citation performance. They found that those disciplines "with more countries in their affiliations performed better in both categories". In other words, the size of the scientific production is related to the sum of all countries' researchers working on a particular area or field which represents the total world production in that field, as observed by Bornmann et al. (2012). On the other hand, other indicators, such as individual counting of articles, total citations, the impact and international cooperation level, as well as other qualitative science-derived issues are dependent of other unrelated factors. Of particular interests in this aspect is the proportion of collaborative articles resulting from co-authorship among researchers from different countries, especially in the case of countries with a small number of published articles, which reflects in an insignificant quantitative and, even less, qualitative contribution to the world scientific output.

Concerning the contribution of the 217 countries to the world output, one can find that by counting the number of documents country by country there is an 1.33 -fold increase in the number of counted articles as compared to the amount of documents effectively published by the whole world. The difference accounts for the double-counting effect due to publications produced in co-authorship by authors from two or more countries. In such cases, documents produced by each country in collaboration with other countries will be counted at least twice. As pointed before (Beys-da-Silva et al. 2014) the double-counting trait is derived from the fact that whenever a publication includes cooperation between or among researchers linked to two or more institutions, countries or fields of knowledge, these articles will be counted more than once (Almeida and Guimarães 2013). Doublecounting is inherent to the bibliometric measures of scientific production of the countries, 
research fields and institutions. In our study we identify it as a trait related to high number of articles resulting from international collaboration, especially from the less productive countries with the more developed ones.

Here we show that international collaboration may have an unconstructive influence affecting directly the usefulness of the scientific impact when such collaboration involves countries with very small number or articles and thus a low scientific output. Actually, a great number of countries still present a very low scientific out put, and the doublecounting feature "masks" the real importance of their genuine contribution to the advance of the world science and may hinder the development of each of these countries. Thus, studies ignoring the factors affecting bibliometric indicators, such as unbalanced scientific collaboration and contribution of double-counting to high impact, often induce a biased analysis of the relevance to the world of countries with lower scientific output. In fact this is a common fault that must be avoided when such scientometric analyses are made. Thus, it is important to know the dimensions of these distortions because they can and in fact, they do introduce bias in the bibliometric analysis of science output, especially when comparing countries and subject areas of research. In this work we compare the data of both indicators, that is to say, with and without considering double-counting.

An expected benefit of international collaboration among countries could be attributed to the expectation of knowledge transfer (Katz and Martin 1997), actually as mention by the authors "Collaboration is one way of transferring new knowledge, especially tacit knowledge". However as shown here, this seems not to be occurring in the cases of unbalanced collaboration as identified in our study. Although several studies dealing with international collaboration have been published little attention has been given to the deleterious effect of excessive co-authorships among researchers from the most developed countries and that of authors from undeveloped countries.

In the present work we aimed to explore the deleterious influence of unbalanced levels of international collaboration to the world's scientific indicators. Such problem expressed as double-counting of articles, involves a large number of countries with very little proper and autonomous scientific production. The observed situation creates an illusory level of scientific performance, which resulted from the unbalanced collaboration between authors from these countries with the authors from more productive ones. Another aspect concerning science development is how it relates to long term prosperity, welfare and competitiveness. This study also shows some relationships implicating these indicators.

\section{Methodology}

In this work we used the qualitative and quantitative bibliometric indicators with the purpose of evaluation of the contribution of the countries in the world scientific production. The InCites' Data Base (Thomson Reuters, Philadelphia, USA, available online through the Portal of Periodicals offered by Fundação Coordenação de Aperfeiçoamento de Pessoal de Nível Superior, CAPES, Brazil) was used as the source of scientific data. The InCites' Data Base provides scientific information of countries, institutions, and individuals as well as to the various areas of knowledge. This extensive Data Base allows its use for both quantitative and qualitative assessment of information covering all scientific areas, including the humanities and social sciences. Here the data of publications in referred journals, including: Number of published documents, Percentage of World Articles, Citations, Impact, International Collaboration Index (ICI) and the Impact Relative to 
World (IRW), were extracted. Data analysis allowed the acquirement of each country's productivity index, enabling comparisons on the national and international levels.

The specific data concerning scientific production used in this analysis were obtained by assessing in the InCites' Data Base: (1) Essential Science Indicators (ESI) composed of 22 large areas covering in brief, all the major scientific fields; (2) Web of Science (WoS) containing 251 categories of sub-areas, fields or research themes derived from the 22 scientific great areas of ESI. In both cases, data from Social Sciences and Humanities were included. The number of documents corresponding to the total of articles, conference papers and reviews published in the 5-year period 2010-2014 were extracted for further analysis. Data were obtained from the Global Comparisons Option-National Comparison Report, designed to measure the performance of the countries and institutions in selected areas of research. The search of all data were reviewed and analyzed from May to November 2015. Counting the number of publications from countries was made carefully taking in consideration the problems arising when such scores are searched (Gauffriau et al. 2007; Larsen and Von Ins 2010; Beys-da-Silva et al. 2014). These problems are related to the occurrence of double-counting of scientific literature, when comparing the production of different countries, institutions and/or fields of knowledge. This is due to the origin of each publication when it results from the cooperation between researchers connected to two or more countries, institutions or different areas. In these cases, the article will be counted more than once.

The bibliometric indicators used in this study were extracted or calculate from InCites' Data Base as described below:

(a) Countries distribution: The 217 countries were divided in three groups according to their scientific contribution to the word's total in the period 2010-2014: Group I includes the countries that have produced individually at least $1 \%$ of the word's articles in the period; Group II is composed of other countries each one producing from 0.1 to $0.99 \%$ of the world sum; Group III is composed of countries with $<0.1 \%$ individual contribution to the world total.

(b) Number of documents: Sum of the number of documents published as full articles, conference papers and reviews of a selected area or country. These documents are relevant items published in a scientific journal covered by the Journal Citation Reports (JCR of Thomson Reuters). This study does not include Editorials, letters, news and meeting summaries, also available in the base, because usually such documents are not cited. In the text and tables we use the words articles or documents with the same meaning.

(c) World percent publications: Number of documents published in a subject area or country divided by the total number of documents in the world. Unless otherwise informed this indicator was obtained from the InCites' Data Base, and it is calculated based on the number of publications without double-counting.

(d) Impact: Number of citations received by articles published by a country, institution, area of knowledge, or a researcher, divided by the total number of papers published in the same period. Here again the data was obtained from the InCites' Data base. The number of citations received by an article indirectly denote the impact caused by the article over the research field and thus it is an indicator of the article's quality.

(e) Impact relative to world (IRW): It concerns to the impact of an area or country relative to the world's average impact of that area or the average of all countries together. An IRW index greater than 1.0 indicates that the impact in a specific area or country is larger than the average scientific impact of all areas together and, in the 
case of countries it means that the impact of a country is higher than the average of all countries.

(f) Double-Counting Index (DCI): This index represents the percent of double counted articles, calculated for each area or field of knowledge. In the case of the world publications it derives from the calculated sum of publications counted country by country, for which the documents published by authors from more than one country will be counted two or more times, depending on the number of foreign countries involved in the partnership. The concept was extracted from the InCites Indicators Handbook which states that "internationally collaborative document is an indicator that only takes into account if a document is international (two or more countries) or not. It does not take into account the total number of countries represented in the publication".

(g) Percentage of international collaboration: The data was obtained from InCites' Data Base; it corresponds to the percentage of articles published by authors belonging to institutions of two or more countries. Since the characterization of an article as having international collaboration depends upon the indication of authors' addresses, we based our study exclusively on the percentage of international collaboration informed by InCites.

The sources of other indicators used in this study are described below:

(h) Human Development Index (HDI): PNUD 2014 http://www.pnud.org.br/IDH/DH. aspx

(i) Percentage of GNP applied in R\&D: 2015 World development indicators: science and technology. United Nations Educational and Cultural Organization (UNESCO) Institute for Statistics http://wdi.worldbank.org/table/5.13

(j) Number of scientists and engineers per million inhabitants: 2015 World development indicators: science and technology. United Nations Educational and Cultural Organization (UNESCO) Institute for Statistics

http://wdi.worldbank.org/table/5.13

\section{Results and discussion}

\section{Double-counting effect in the world' scientific production}

In the period 2010-2014, the 217 countries listed in the Thomson Reuters' InCites Data Base produced a total of 6,811,602 articles. However, considering the individual country by country contribution, the sum reaches a total of 9,064,873 articles (Table 1). The difference accounts for the double-counting effect due to publications produced in coauthorship by authors from two or more countries. Here the additional counting of 2,253,271 articles represents an increase of $33.1 \%$ in the amount of articles listed. In this work, counted articles for each country includes the publications resulting from international collaboration. Unfortunately due to the large numbers of articles and countries in this study, fractional counting as recommended by Gaufrial and Larsen 2005, could not be applied here. As pointed above, the double-counting trait is derived from the fact that whenever a publication includes cooperation between or among researchers linked to two or more institutions, countries or fields of knowledge, the article will be counted more than once. Double-counting is inherent to the bibliometric measures of scientific production of the countries, research fields and institutions because of the steady increase of international 
Table 1 World scientific production in the 22 great ESI areas: 2010-2014. The double-counting effect Source: InCitesTM, Thomson Reuters. Report created: 12/05/2015. Data source: Web of science

\begin{tabular}{llllllc}
\hline Nr. & Subject area & $\begin{array}{l}\text { Number } \\
\text { of } \\
\text { countries }\end{array}$ & $\begin{array}{l}\text { No double } \\
\text { counting } \\
(\mathrm{A})\end{array}$ & $\begin{array}{l}\text { With double } \\
\text { counting } \\
(\mathrm{B})\end{array}$ & $\begin{array}{l}\text { Difference } \\
(\mathrm{B}-\mathrm{A}) \\
(\mathrm{C})\end{array}$ & $\begin{array}{l}\text { \% Double } \\
\text { counting (C/ } \\
\text { A) } \times 100\end{array}$ \\
\hline 1 & Agricultural sciences & 193 & 190.460 & 240.348 & 49.888 & 26.2 \\
2 & Biology and Biochemistry & 190 & 338.993 & 443.237 & 104.244 & 30.8 \\
3 & Chemistry & 184 & 750.403 & 932.289 & 181.886 & 24.2 \\
4 & Clinical medicine & 206 & $1,245,135$ & $1,608,227$ & 363.092 & 29.2 \\
5 & Computer science & 148 & 157.999 & 212.953 & 54.954 & 34.8 \\
6 & Economics and business & 174 & 121.532 & 166.544 & 45.012 & 37.0 \\
7 & Engineering & 183 & 541.591 & 679.321 & 137.730 & 25.4 \\
8 & Environment/ecology & 202 & 204.367 & 293.293 & 88.926 & 43.5 \\
9 & Geosciences & 204 & 197.908 & 305.373 & 107.465 & 54.3 \\
10 & Immunology & 202 & 118.099 & 174.626 & 56.527 & 47.9 \\
11 & Materials science & 163 & 340.452 & 425.450 & 84.998 & 25.0 \\
12 & Mathematics & 165 & 197.820 & 262.022 & 64.202 & 32.5 \\
13 & Microbiology & 191 & 96.090 & 133.333 & 37.243 & 38.8 \\
14 & Molecular biology and & 186 & 205.993 & 299.111 & 93.118 & 45.2 \\
& genetics & & & & & \\
15 & Multidisciplinary & 159 & 13.396 & 20.858 & 7.462 & 55.7 \\
16 & Neuroscience and behavior & 174 & 239.632 & 323.814 & 84.182 & 35.1 \\
17 & Pharmacology and toxicology & 183 & 179.360 & 228.197 & 48.837 & 27.2 \\
18 & Physics & 172 & 551.015 & 820.847 & 269.832 & 49.0 \\
19 & Plant and animal science & 207 & 335.816 & 463.507 & 127.691 & 38.0 \\
20 & Psychiatry/psychology & 181 & 182.819 & 238.278 & 55.459 & 30.3 \\
21 & Social sciences, General & 208 & 405.852 & 501.462 & 95.610 & 23.6 \\
22 & Space science & 134 & 68.302 & 153.882 & 85.580 & 125.3 \\
World & 217 & $6,811,602$ & $9,064,873$ & $2,253,271$ & 33.1 \\
\hline & & & & & &
\end{tabular}

scientific collaboration. In our study, this feature appears in the high number of articles resulting from international collaboration especially from the less productive countries together with more developed ones (see below). Thus, it is important to know its dimension because it can and in fact, it does introduce bias in the scientometric analysis of science output, especially in cases of comparisons of countries and subject areas of research. In this work we compare the data of both indicators, that is to say, with and without doublecounting.

\section{Double-counting effect in the scientific fields}

Table 1 lists the world scientific production in the 22 great areas of ESI (Thomson Reuters Data Base) in the 5 years period 2010-2014. As mentioned above, 217 countries are engaged in scientific research and publication worldwide. The question arisen from this information is: What is the contribution of each country to the production of new knowledge in each of these 22 areas and in more than 200 specific scientific fields? As shown in Table 1 all countries are developing research in the whole spectrum of scientific 
activities. Space Science is the area with the minimal number of publishing countries, 134, whereas Social Sciences congregated the maximal number, 208 countries, publishing in this area. As an average 182 countries are involved in publications of all scientific fields. This means that most countries, no matter how developed they are, produce scientific articles in several areas and specific fields. It is then important to know how much of such production derived from the effort of having a proper system for stimulating scientific development and what fraction represents a casual participation of the countries in these publications. In this context it is important to discriminate types or levels of international scientific cooperation, in which a country can behave as an active or passive agent. Here we show that the quantification of double-counting articles can indirectly indicate the proportion of country's participation in the publications of a particular field. Table 1 presents both indicators: the number of articles with and without double-counting in each ESI area in the 2010-2014 period. The Double-Counting Index was calculated by subject area as described in the methods section and represents the percent ratio between the number of articles with double-counting and that of the articles without double-counting in an individual area. Publications involving 182 countries accounted $33.1 \%$ of doublecounted articles. In some areas such as Space Science for instance, the actual number of articles $(68,302)$ generated 153,882 double-counted documents. The difference $(85,580$ articles) accounts for a Double-Counting Index of $125.3 \%$ which implies that this area attracts an unusual proportion of international collaboration. Besides space science, 11 other areas (multidisciplinary, geosciences, physics, immunology, molecular biology and genetics, environment/ecology, microbiology, plant and animal science, economics \& business, neuroscience $\&$ behavior and computer science) also show high level (above the table average) of double-counted articles. On the other hand the lowest values are found for Social Sciences $(23.6 \%)$, Chemistry (24.2\%), Materials Science $(25.0 \%)$ and Engineering $(25.4 \%)$.

\section{Double-counting effect in the scientific production of countries}

As happen with the observed dispersion of the scientific production among the areas of knowledge seen in Table 1, a similar situation is detected when such distribution is considered for the 217 countries. Table 2 shows the distribution of all 217countries into three different groups, according to their contribution to the world production. Group I includes the most productive countries which individually contributed at least with $1 \%$ for the world total. The 24 countries in Group I represent $11.1 \%$ of the world countries but published 7,605,317 articles or $83.9 \%$ of the total double-counted world scientific production. Group II is composed of 40 other countries and accounts for $18.4 \%$ of the total countries, each one producing from 0.1 to $0.99 \%$ of the world sum, thus making a total of $1,285,850(14.2 \%)$ of the articles' total. Group III is composed of 153 countries $(70.5 \%$ of the total) with $<0.1 \%$ individual contribution, representing altogether 173,706 articles $(1.9 \%)$ of the world scientific output (Table 2). The Table also presents other qualitative characteristics of each country's groups indicating the average data for: the impact, impact relative to world (IRW), percentage of cited articles, International Collaboration Index (ICI), percentage of the Country's GNP applied in R\&D and the proportion of Scientists and Engineers per million inhabitants. As shown in Table 2, several of these qualitative indicators reflected characteristics of the three groups and they will be better explored in the analysis of the country's groups presented below.

Table 3 lists the 24 countries belonging to Group I and presents their scientific output in the period 2010-2014, in all areas and scientific fields of knowledge. Similarly, as 
Table 2 Distribuition of countries according to their individual scientific production: 2010-2014 Source: InCitesTM, Thomson Reuters. Report Created: 12/05/2015. Data source: Web of science

\begin{tabular}{|c|c|c|c|c|}
\hline \multirow[t]{2}{*}{ Indicators } & \multicolumn{3}{|l|}{ Countries distribution } & \multirow{2}{*}{$\begin{array}{l}\text { World } \\
\text { data }\end{array}$} \\
\hline & $\begin{array}{l}\text { Group I } \\
\text { Countries with more } \\
\text { than } 1 \%\end{array}$ & $\begin{array}{l}\text { Group II } \\
\text { Countries with } 0.1 \\
\text { to } 0.99 \%\end{array}$ & $\begin{array}{l}\text { Group III } \\
\text { Countries with } \\
<0.1 \%\end{array}$ & \\
\hline Number of countries & 24 & 40 & 153 & 217 \\
\hline Countries $\%$ of world & 11.1 & 18.4 & 70.5 & 100 \\
\hline Average HDI & 0.844 & 0.782 & 0.631 & 0.686 \\
\hline $\begin{array}{l}\text { Total Articles with double- } \\
\text { counting }\end{array}$ & $7,605,317$ & $1,285,850$ & 173.706 & $9,064,873$ \\
\hline $\begin{array}{l}\text { Total articles without double- } \\
\text { counting }\end{array}$ & - & - & - & $6,811,602$ \\
\hline$\%$ World production & 83.9 & 14.2 & 1.9 & 100 \\
\hline $\begin{array}{l}\text { International collaboration: } \\
\text { percentual average }\end{array}$ & 43.0 & 55.8 & 85.2 & 60.2 \\
\hline$\%$ Cited articles & 68.8 & 64.2 & 64.9 & 65.2 \\
\hline Average of impact factor & 6.4 & 5.3 & 4.8 & 5.1 \\
\hline Impact realtive to world (IRW) & 1.2 & 1.0 & 0.9 & 1.0 \\
\hline \%GNP applied in R\&D & 2.0 & 1.1 & 0.3 & 2.2 \\
\hline $\begin{array}{l}\text { Scientists and engineers per } \\
\text { million inhabitants }\end{array}$ & 3.529 & 2.288 & 510 & 1.268 \\
\hline
\end{tabular}

Distribution based on the world \% of scientific production of each individual country considering the double counting of articles

compared to Table 1, the countries of Group I produced most publications of the total computed for each of the 22 great areas (data not shown). Thus, in this work we named Group I as the most productive countries. Table 3 presents, besides the quantitative data of the countries, their Human Development Index (HDI). The Impact of the publications from these 24 countries varies from 9.8 (Switzerland) to 3.1 (Russia and Turkey) with an average index of 6.4 which is substantially higher than that of the world's average (5.1).The International Collaboration Index (ICI) of countries in Group I varies from $69.1 \%$ (Switzerland) to $20.0 \%$ (Turkey) being the average $(43.0 \%$ ) much smaller than the world's average $(60.2 \%)$. This last value is highly influenced by the high proportion of international collaboration (85.2\%) of the countries in Group III (Table 2). Actually in Group I only four countries (Switzerland, Belgium, Denmark and Sweden)have ICI higher than the world's average. As compared to countries with very high impact consequent to an extremely high ICI (see Table 6), countries of Group I, though having relatively high scientific impact, do not show such a strong correlation with ICI. In fact several countries of this group show the lowest ICI values among all countries. This indicates that some countries of Group I, due to their scientific leadership, possess and actively offer attractive conditions for international cooperation with other countries. Due to their sizeable scientific production, this condition does not affect significantly their ICI. On the other hand, this situation works the other way around for the countries with very small scientific production, as those of Group III. Another central aspect to be noticed is that the majority of the countries of Group I also occupy the highest level of economic and social 


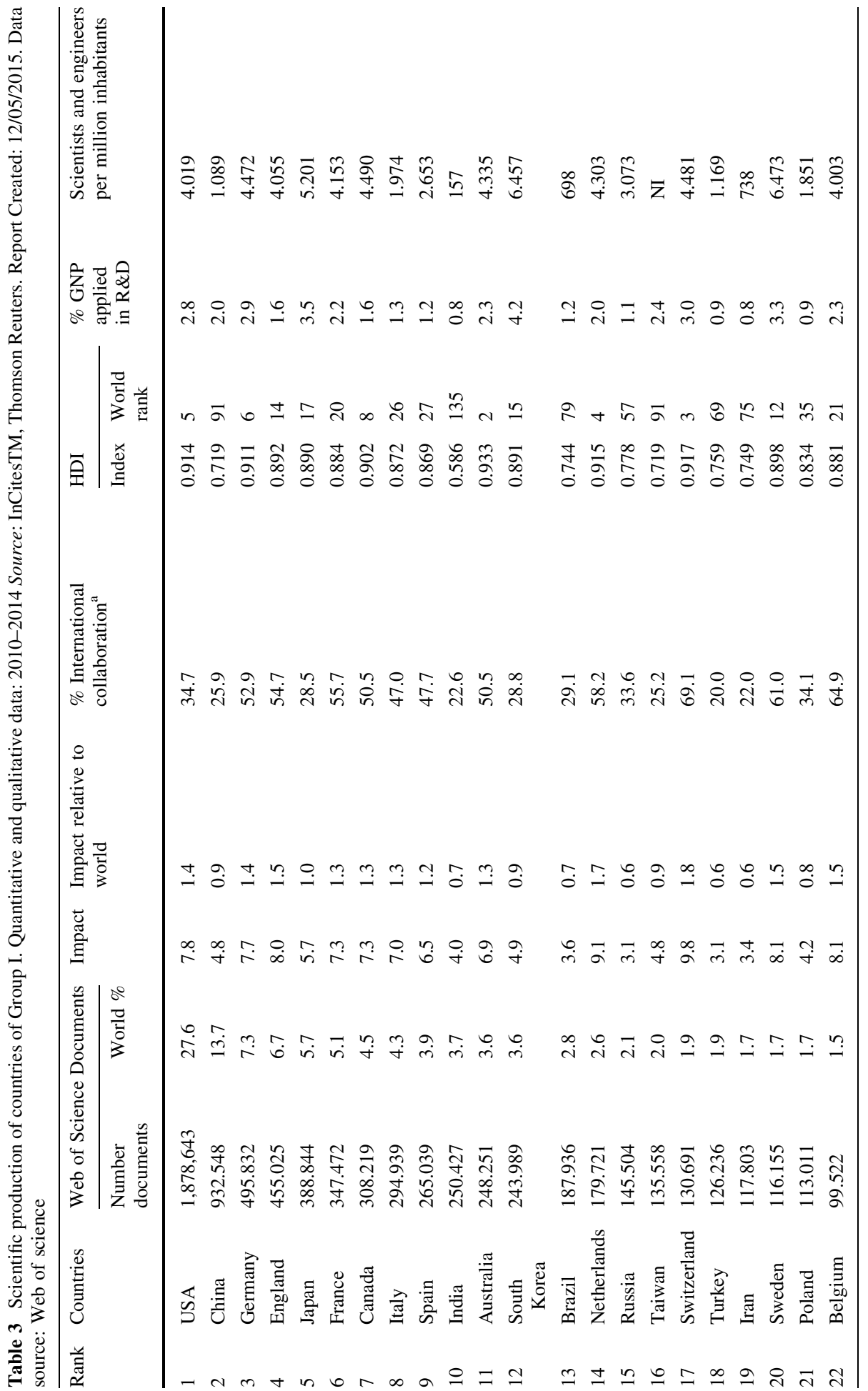




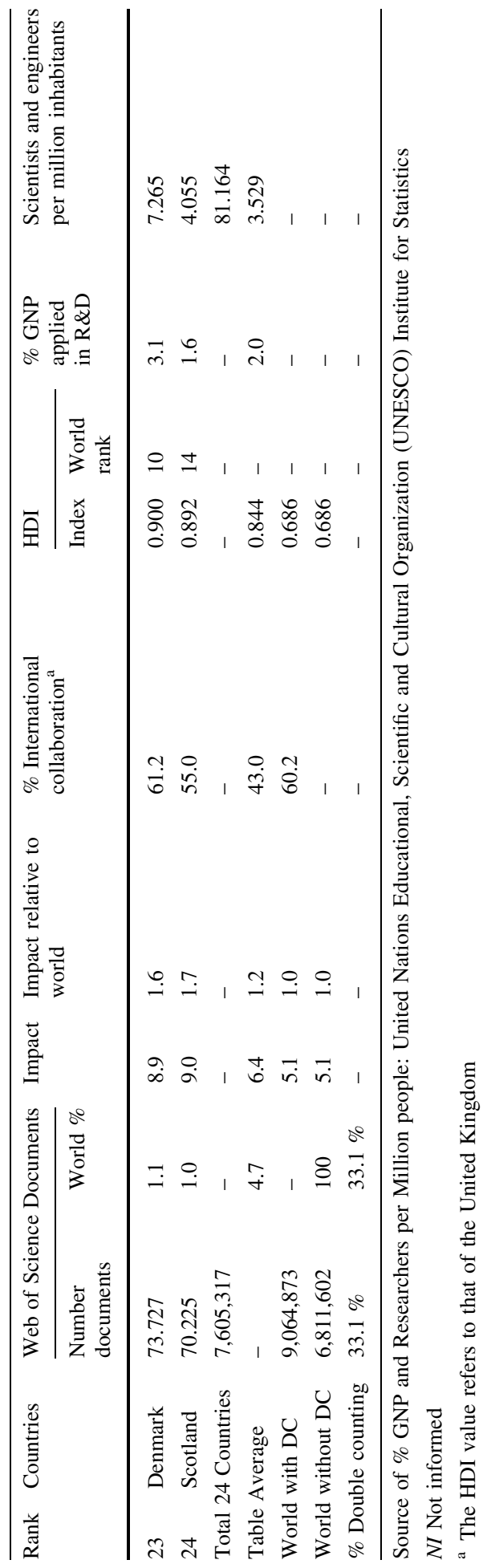


development, what can be depicted from their Human Development Index (HDI). It is noticeable that the countries of Group I present a high average HDI (0.844), which is much higher than the world's data. In fact, only seven countries (India, Taiwan, China, Brazil, Iran, Turkey and Russia) show index values below this level, with a variation from 0.586 (India) to 0.933 (Australia).

Actually the countries of Group I are among the most developed ones, thus indicating the importance of science as an instrument to improve and support social and economic welfare. As mentioned by Jefrey Wadworth (2014): "Fortunately, there is ample historical evidence that research and innovation contribute, in the short and long terms, to prosperity and competitiveness, as well as to the resolution of society's greatest challenges in areas like health, energy and security". Unfortunately this expectation seems not to be happing with countries that can only offer some desirable advantages for cooperation, such as huge and attractive biodiversity for instance. It is also true that the countries of Group I are investing the highest proportion of their GNP in R\&D and also, at the same time, possess a comfortable condition of having high number of scientists and engineers (Table 3). In fact the countries of this group apply more than $0.8 \%$ (average $2.0 \%$ ) of their GNP in S\&T and, with exception of India, they possess a high proportion (average 3529) of scientists and engineers per million inhabitants. Putting together all the quantitative and qualitative indicators, the great majority of countries in Group I constitute a kind of first league of scientific development.

Table 4 lists the 40 countries pertaining to Group II and shows their scientific output in the period 2010-2014. Together these countries produced 1,285,850 articles (14.2\% of the world's total) also covering all areas and scientific fields of knowledge. The scientific qualitative data of Group II is summarized in Table 4. The scientific impact of the 40 countries varies from 2.8 (Nigeria) to 8.4 (Singapore) with an average index of 5.3, not too far from that of the world's average (5.1). Their IRW average index is 1.0 (range 0.5 to 1.5), with several countries showing IRW values in the same range as those of countries of Group I. With few exceptions, most countries of this group show intermediate range of international collaboration. As shown in Table 4, the International Collaboration Index (ICI) of these countries varies from $37.0 \%$ (Serbia) to $87.2 \%$ (Kenya), with an average $(55.8 \%)$ smaller than the world's average $(60.2 \%)$ but much smaller than that of Group III $(85.2 \%)$. Similarly to Group I, some countries of this group can also exert attractive influence for active international cooperation. Concerning to the Human Development Index, the countries of Group II present a HDI average of 0.782 varying from 0.504 (Nigeria) to 0.944 (Norway). Related to the percentage of GNP invested in R\&D a mean index of $1.0 \%$ was found for Group II countries. However one can see in the table that there is a great dispersion (range from $0.1 \%$ for Indonesia to $4.2 \%$, Israel) of such indicator among the countries of this group. In fact about one third of these countries invest more than $1 \%$ of their GNP in R\&D. Actually these are the countries that share with others of Group I high social and economic standard of life, as also indicated by their HDI.

Besides the quantitative data presented by countries of Groups I and II, Tables 3 and 4 also show the qualitative indicators listed before. In addition, other important characteristics of the countries' indicators related to scientific production, such as the Human Development Index (HDI), the percentage of GNP applied to R\&D and the proportion of scientists and engineers related to the size of population, are indicated in the tables. Thus, taken together the qualitative data of Groups I and II could result in another kind of raking (Table 5) when the Impact index is used in order to classify the countries. Concerning specially to impact, HDI, and other qualitative indicators, the ranking of countries in Groups I and II shows several changes in positions. In fact, some countries of Group II 


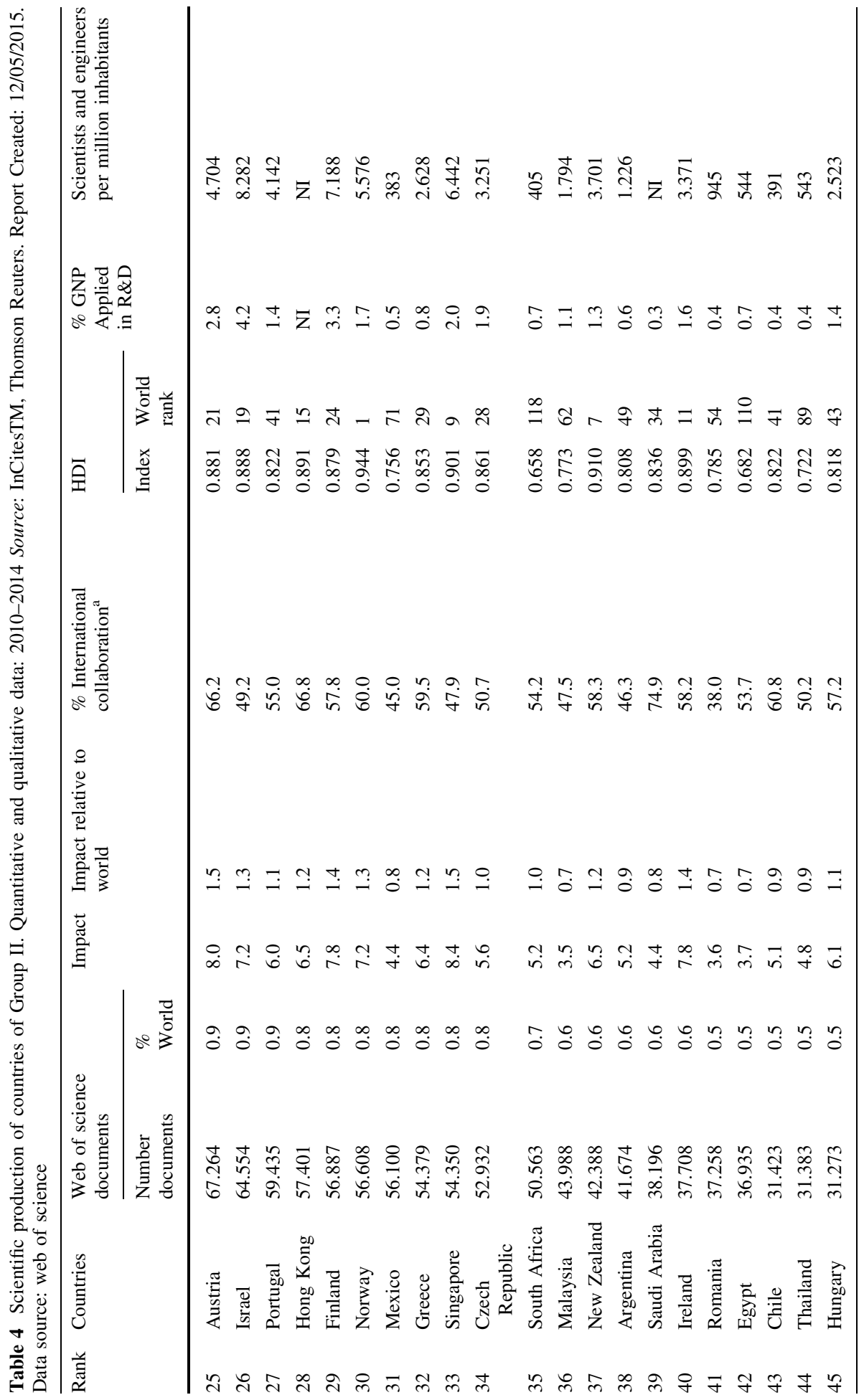




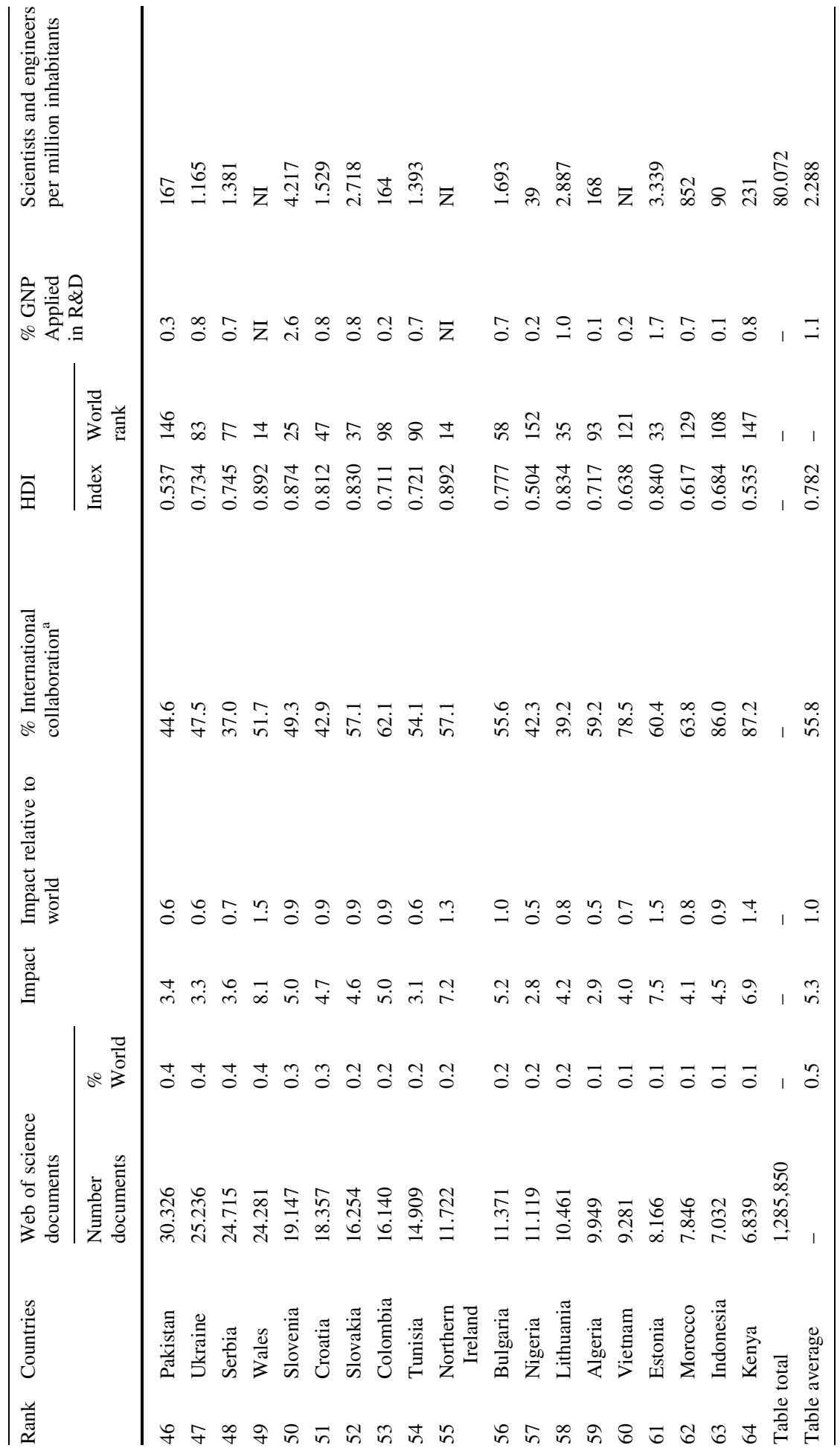




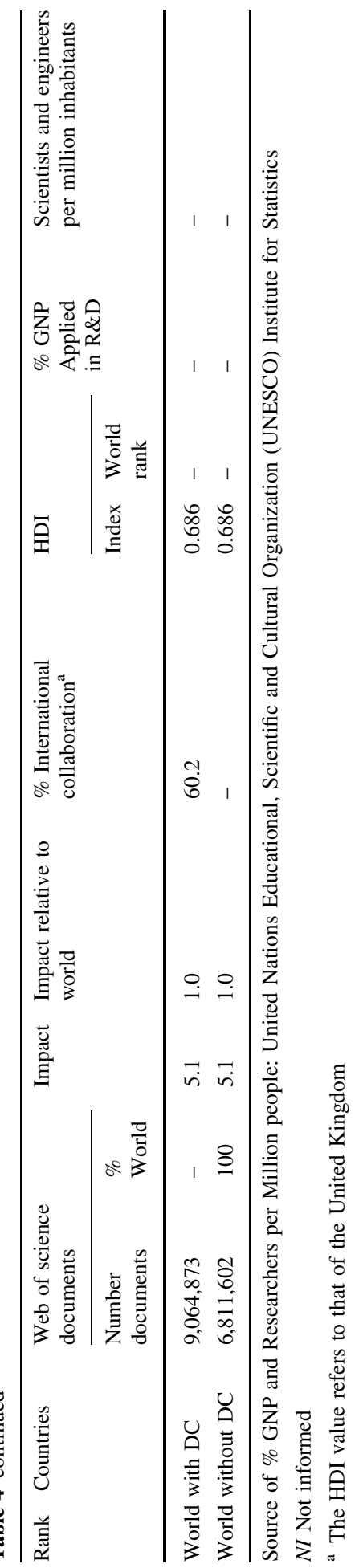


have qualitative data similar to those in Group I, while among the most productive countries several ones do not meet the same outstanding level of this group. This confirms the lack of correlation between number of publications and impact of articles (not shown). Such differences can be seen in Table 5 .

Group III is composed of 153 countries (70.5\% of the total) each one publishing $<0.1 \%$ of the world's production, representing altogether 173,706 articles (1.9\%) of the world scientific output (Table 2). In this group, 74 countries (48\%) produced together in the period 2010-2014 a sum of 9009 articles, about $0.1 \%$ of the world's total (data not shown). In the 5 year period, 36 countries in this group published $<100$ articles each. On the other hand, a particular feature of Group III is the high percentage of international collaboration presented by most of its components. Among the 153 countries belonging to this group, only 15 countries had an international scientific collaboration of $<65 \%$ and none below $51 \%$. Therefore, an unexpected average of $85.2 \%$ is found for the international collaboration indicator of the countries of this group (Table 2). In fact, the great majority of countries in Group III display much higher individual levels of international scientific collaboration than countries in groups I or II. As a consequence, the Impact index's average (4.8) of Group III is very close to the world's average (5.1) which is heavily influenced by this group of countries. Actually, as shown in Table 6, several countries of this group have the highest values of scientific Impact among all countries. Considering this distortion, one should take into account as inadequate the usual comparison of country's impact versus the world impact.

Table 6 lists 20 countries for which the Impact exceeds the world's average in values above 1.5 IRW, i.e. countries with Impact value of 8.0 or higher. As shown in the table, ten of these countries (Vatican, Bermuda, Iceland, Mozambique, Republic of Georgia, Equatorial Guinea, Gambia, Panama, Armenia and Monaco) belong to Group III (Table 2), showing IRW values from 1.6 to 2.5. Actually, this group includes the five countries with the highest Impact in the world. Another feature of these ten countries is their very small number of publications: 14,537 articles altogether or $<0.2 \%$ of the world's total in the 5 years period, but with a very high Impact average (9.1). Additionally an extremely high international collaboration index (average of $88.2 \%$ ) is characteristic of these countries.

The distortions seen in Table 6 can be better stressed when comparing the Impact data of the countries related to their scientific production accounted area by area of the InCites Data Base. Table 7 illustrates a comparative data of the Impact values of articles published in the InCites' 22 areas of knowledge distributed by the different countries. It shows that the same distortions seen in Table 6 are even more expressive here. The table depicts the ten highest Impact values presented by countries relative to publications in each of the 22 areas. It can be seen that rarely any country of Groups I or II occupies higher places in this chart. In contrast, among the 220 positions existing in Table 7 , the great majority (168/220 or $76 \%$ of the positions) are occupied by countries belonging to Group III. These countries show very high IF values in several areas: Multidisciplinary, Computer Science, Molecular Biology and Genetics, Physics, Space Science, Biology \& Biochemistry, Engineering and Clinical Medicine. On the other hand, only eight Group I countries (Scotland, Switzerland, Netherlands, Denmark, England, USA, Canada and France) appear 25 times (11.4\%) in the chart, usually with much lower IF values. Concerning to Group II, 12 countries (Wales, Ireland, Singapore, Hong Kong, Finland, Bulgaria, Croatia, Ukraine, Colombia, Estonia, Kenya and Serbia) occupy 27 positions $(12.3 \%)$ out of the 220 possibilities. 


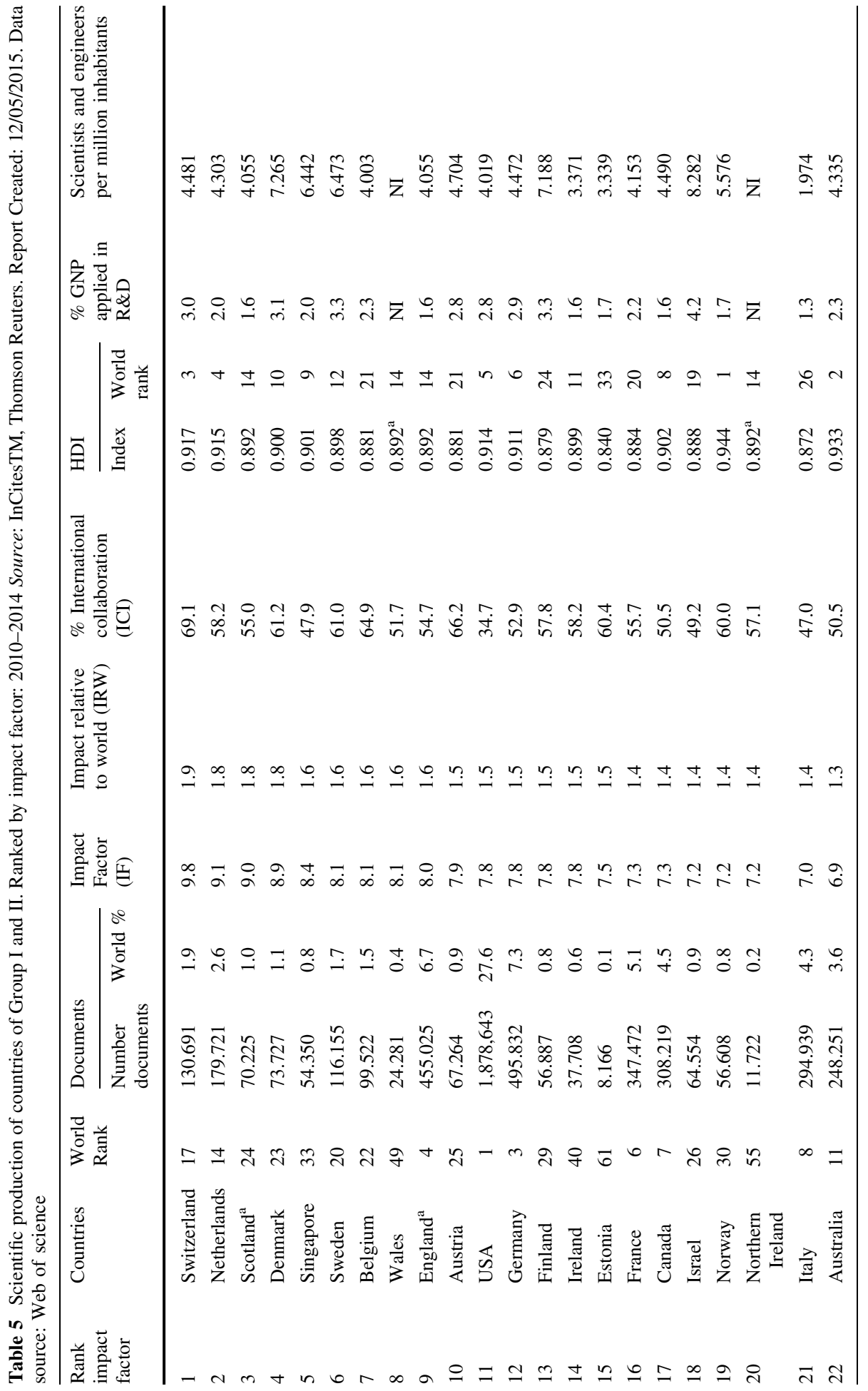




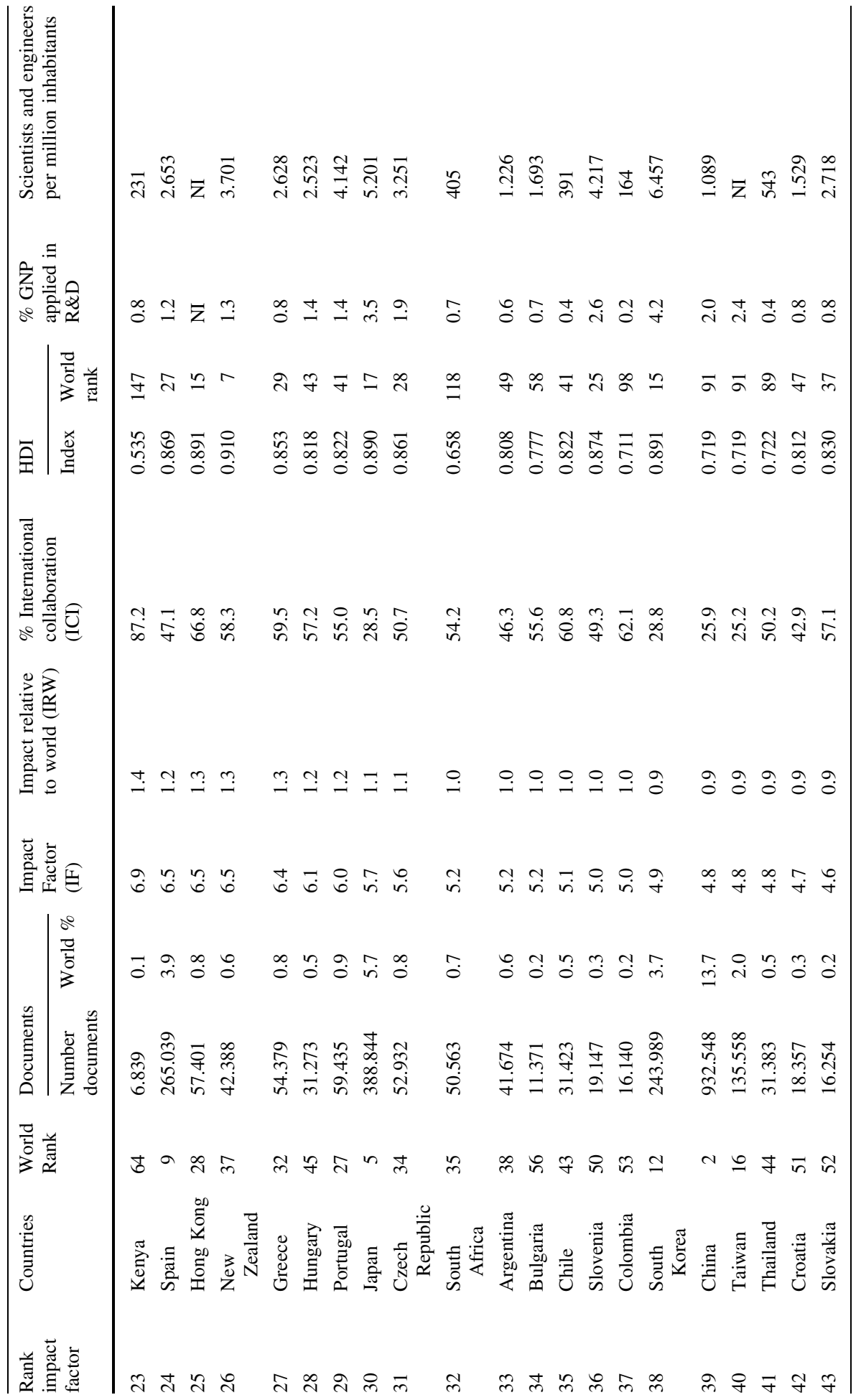




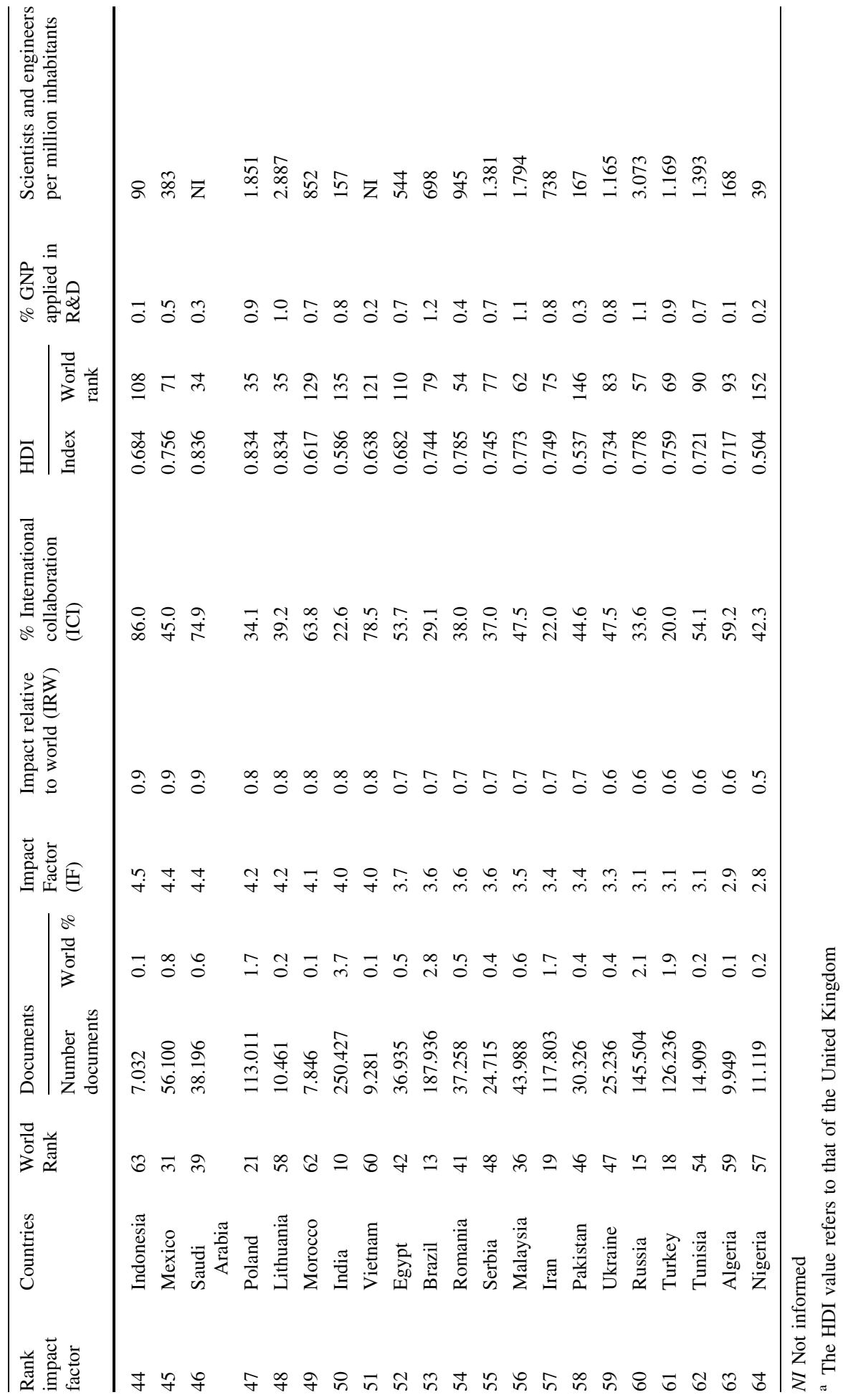




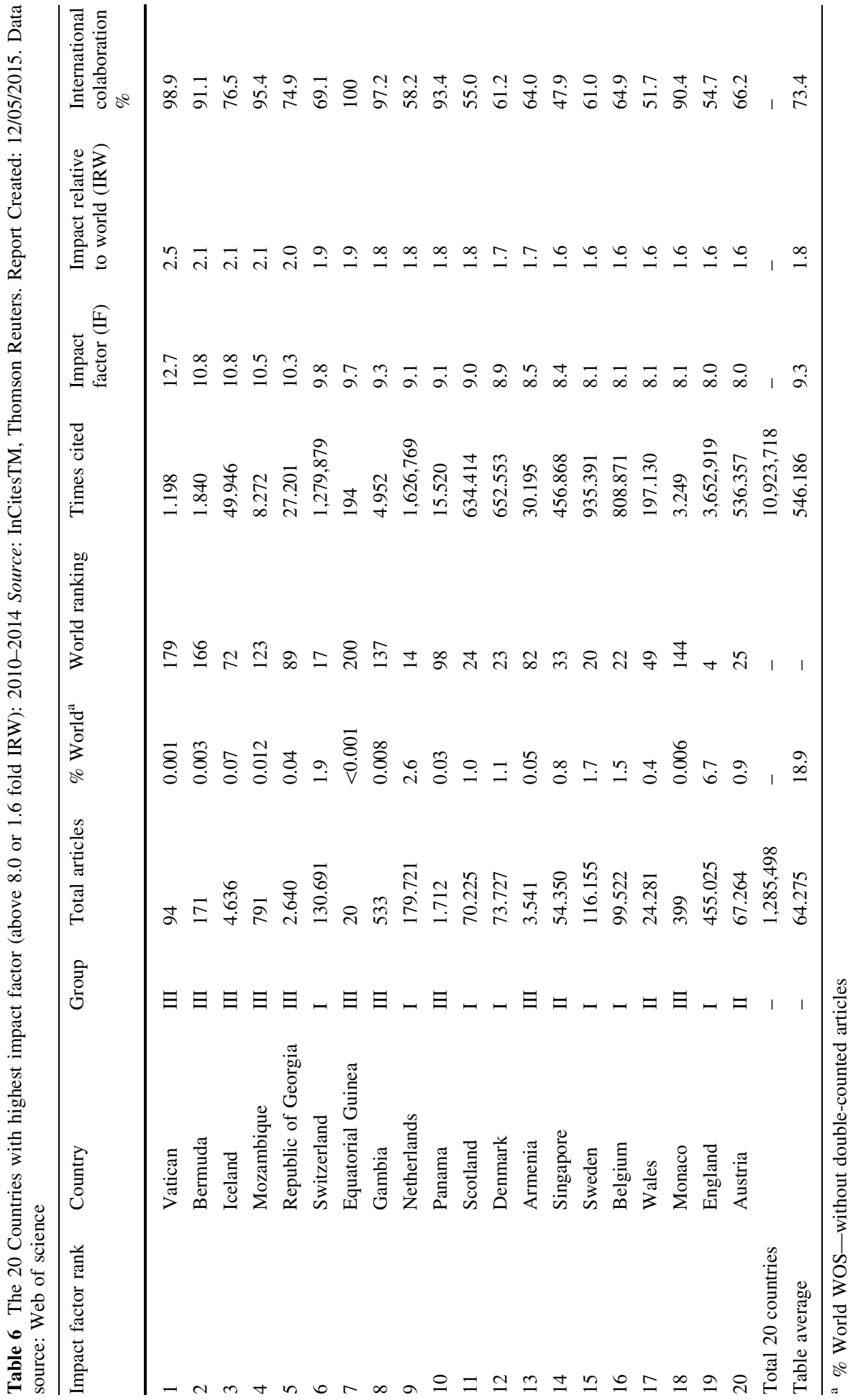




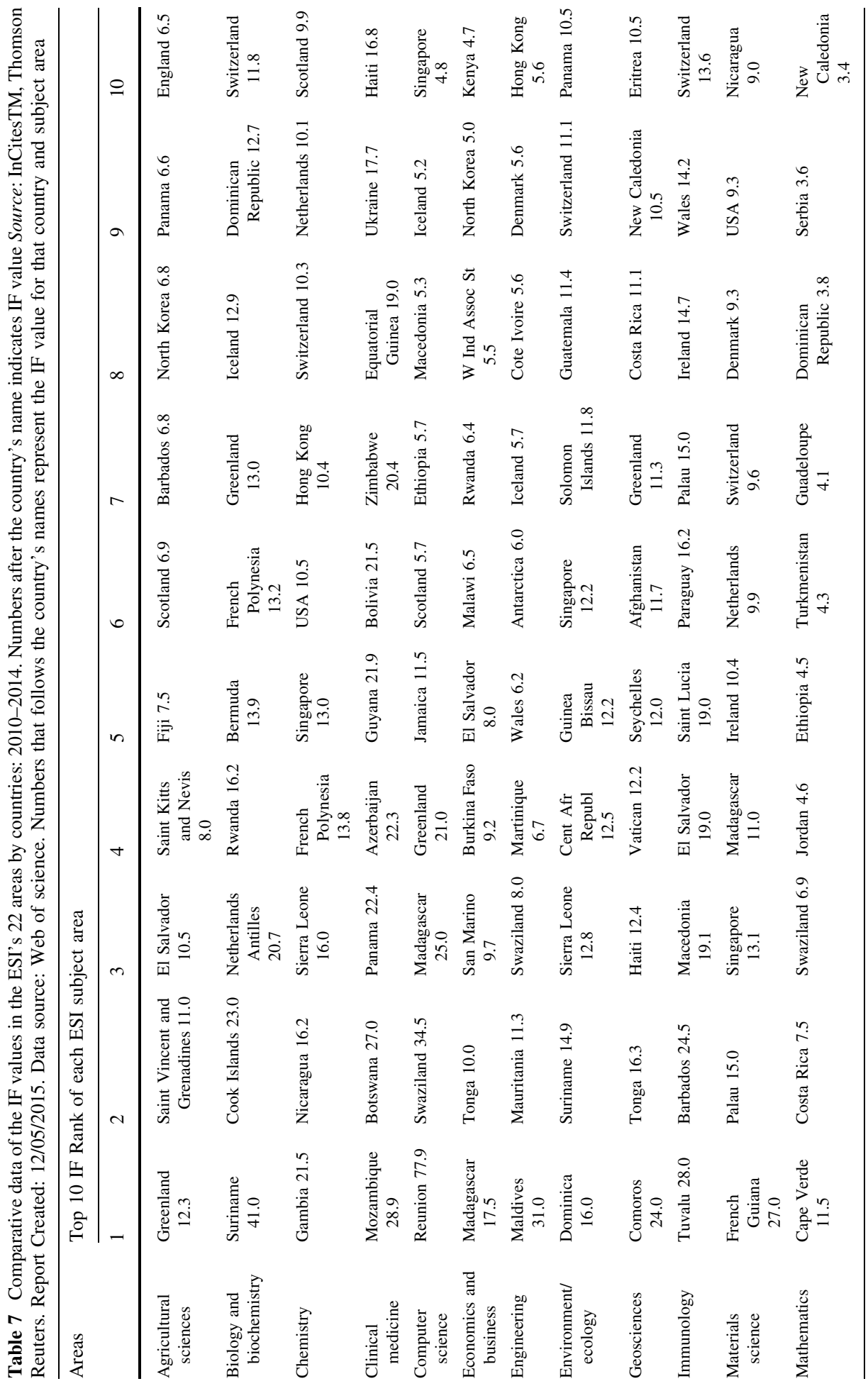




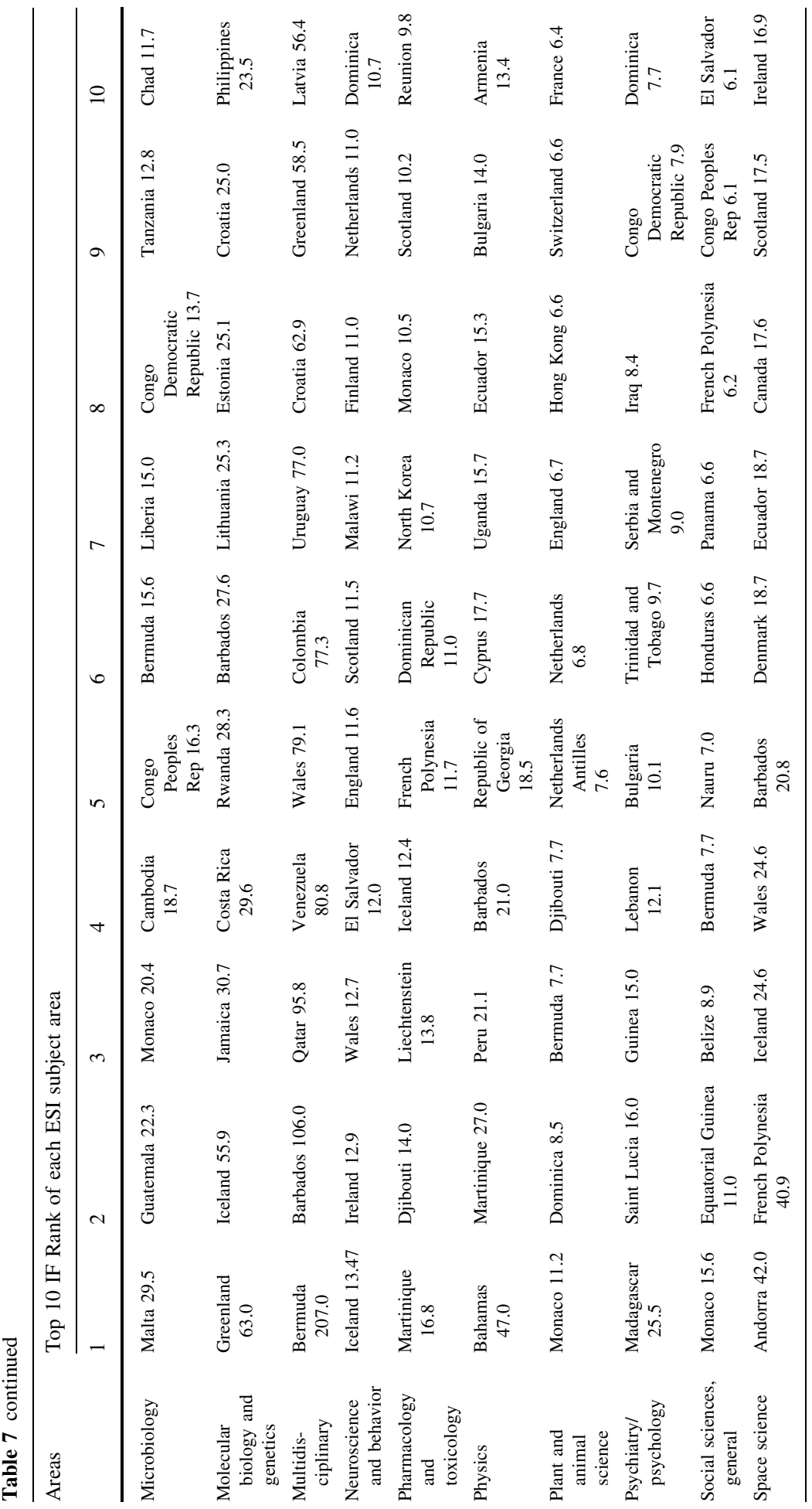




\section{Concluding remarks}

In this study, to our knowledge the first of its kind, we analyzed the effect of the unbalanced international collaboration as cause of misleading information on each country's contribution to the scientific world output. The influence of international collaboration on the scientific production of the 217 active countries of the world for the 5 years period, 2010-2014 was analyzed. The importance of international collaboration is highly appreciated and a "Global Science Engagement" has been recently suggested by Geraldine Richmond in Science (Richmond 2016) in order to confront world challenges. Several countries including South Korea, Australia, China, Taiwan, Iran, and others, have been exploring this idea, despite they have started financing science research much later than the traditional countries. However, this encouragement towards collaboration implies the need for a more participative contribution of all partners in confronting challenges such as food quality, water disposition, healthy insurance, energy supply and security, all kinds of world problems requiring a scientific approach to be solved. In fact, as suggested by the Australians (Australian Academy of Science 2010, page 2), it must be assumed by the countries that "to meet national needs and assist national ambitious it requires increased strategic focus and commensurate support". This applies especially to the less developed countries as seen with the BRICS, for instance (Finardi 2015).

In this work we show how International Collaboration implicates in a high percentage (ca $33 \%$ ) of double-counted world articles thus impacting several qualitative data such as citations, the scientific Impact and the Impact Relative to World (IRW). In addition we explore the possible influence of some indirect indicators such as the Human Development Index (HDI), as they relate to the scientific qualifications of the countries. The main concern raised is relative to the countries of Group III. From the analysis of the indicators presented here it can be concluded that the scientific output of the countries in Group III, represented by articles produced by themselves or in symmetric or balanced international collaboration, is highly insignificant. The great number of countries in this group $(70.5 \%$ of the whole world) is characterized by a very poor contribution to the world scientific production, though associated to an extraordinary high proportion of international collaboration, resulting in a distortive proportion of artificially qualified articles. In fact it can be assumed that most countries of Group III, as well as some ones in Group II, particularly due to the small size of their scientific community may function more passively in such collaborative effort. Collaborations in such cases appear to happen because of the existence of some unique and attractive subjects to be scientifically explored, usually restricted to some specific fields of research, very particular of these countries.

The very small number of individual articles of these countries are usually published with highly productive authors from the most developed countries, which in turn receive a great number of citations. Several authors confirmed this. As a consequence, these countries show high scientific Impact for their few publications (Table 2). Because of the large number of the countries in Group III, a distortion on the average Impact of the whole world is seen. Actually, this level of distortion is even more evident when analyzing individual areas and scientific fields (Tables 6 and 7). In contrast, Tables 3 and 4 show that 14 out of 64 countries, of which only 4 are in Group I (Switzerland, Sweden, Belgium and Denmark) and 10 others ones in Group II (Austria, Hong Kong, Saudi Arabia, Chile, Colombia, Vietnam, Estonia, Morocco, Indonesia and Kenya) show International Collaboration Index (ICI) above $60 \%$ with mean averages for the groups of 43.0 and $55.8 \%$, respectively. For comparison, the great majority of countries of Group III present very high ICI, with an 
average of $85.2 \%$, thus revealing their high proportion of double-counted data. On the other hand, these countries producing very small number of articles have the greatest individual scientific Impact, resulting from intense International Collaboration, usually with the most developed countries, which implies that the articles originated from such collaboration usually receive a high number of citations. As indicated by Smith et al. 2014, in general, independently of the number of publications, high levels of international collaboration usually produce better journal placement and great number of citations and thus higher Impact. When applied to countries with very small number of publications, this trend induces a biased view of the country's productivity and of its own qualitative contribution to the world production. In a recent review, Tahamtan et al. (2016) analyzing the factors which affect scientific citations, found that, besides other factors, "authors' reputation, authors' academic rank and international cooperation are stronger predictors for citations".

Thus, concerning international attraction for cooperation, here again, the observations made by the Australians for themselves (Australian Academy of Science 2010, page 12), can be applied to the poor countries, in that they should be "more than a source of raw material or a convenient observation platform". Such situation creates a biased view about their real scientific engagement in the effort to improve their development and to help solve the problems of their own countries. Accordingly it is observed that most of these countries present a very poor Human Development Index. Here, one relevant question concerns to the role of passive international cooperation: is it possible that the kind of non-symmetric collaboration exerted by the most developed countries, as seen in these numbers, be appropriate to help these passive countries to overcome their challenges and to create any scientific, social or economic progress? Although UNESCO (UNESCO Science Report 2010) considers the rise of international cooperation as linked to the increasing research capacity of developing countries, this assumption seems not to be the case for these countries. Inversely of what is being done, these countries should take into account that a minimal level of autonomous research is the key base for aspiring future prosperity. Several authors (Bozeman and Corley 2004; Van Raan 1998; May 1997; King 2004) suggest a similar conception.

From the above analysis it is assumed that very high levels of international collaboration, as presented by many countries, deceives the bibliometric indicators regarding the importance of their scientific production and jeopardizes their qualitative outputs. In fact these misleading data distort the analysis of the qualitative output of these countries, artificially increasing the scientific Impact of many of them, and also affecting the Impact of scientific fields of research in which they engage. Thus, it is concluded that when dealing with the qualitative contribution of countries to the world output, one must take in consideration the level of international cooperation because as seen here it can and in fact it does create false impression of the real contribution of countries to their own development.

Acknowledgments The authors acknowledge Dr. Celia R. Carlini for critically reading this manuscript. This work received financial support from the Brazilian agencies: Conselho Nacional de Desenvolvimento Científico e Tecnológico (CNPq, Grant 309371/2011-2) and Fundação de Amparo a Pesquisa do Estado do Rio Grande do Sul (FAPERGS, Grant 2252-2551/14-0).

Open Access This article is distributed under the terms of the Creative Commons Attribution 4.0 International License (http://creativecommons.org/licenses/by/4.0/), which permits unrestricted use, distribution, and reproduction in any medium, provided you give appropriate credit to the original author(s) and the source, provide a link to the Creative Commons license, and indicate if changes were made. 


\section{References}

Abramo, G., D’Angelo, C. A., \& Di Costa, F. (2009). Research collaboration and productivity: Is there correlation? High Education, 57, 155-171.

Akre, O., Barone-Adesi, F., Pettersson, A., Pearce, N., Merletti, F., et al. (2011). Differences in citation rates by country of origin for papers published in top-ranked medical journals: Do they reflect inequalities in access to publication? Journal of Epidemiology Community Health, 65, 119-123.

Almeida, E. C. E., \& Guimarães, J. A. (2013). Brazil's growing production of scientific articles-How are we doing with review articles and other qualitative indicators? Scientometrics, 97, 287-315. doi:10. 1007/s11192-013-0967-y.

Australian Academy of Science (2010). Internalization of Australian Science. Position Paper, Feb 2010. www.science.org.au/reports/index.

Barjack, F., \& Robinson, S. (2007). International collaboration, mobility and team diversity in the life sciences: Impact on research performance. Social Geography Discussion, 3, 121-157.

Beys-da-Silva, W. O., Santi, L., \& Guimarães, J. A. (2014). Mangroves: A threatened ecosystem underutilized as a resource for scientific research. Journal of Sustainable Development, 7, 40-51. doi:10. 5539/jsd.v7n5p40.

Bornmann, L., Schier, H., Marx, W., \& Danell, H. D. (2012). What factors determine citation counts of publications in chemistry besides their quality? Journal of Informetrics, 6, 11-18.

Bozeman, B., \& Corley, E. (2004). Scientists' collaboration strategies: Implications for scientific and technical human capital. Research Policy, 33(4), 599-616.

Finardi, U. (2015). Scientific collaboration between BRICS countries. Scientometrics, 102, 1139-1166.

Frame, J. D., \& Carpenter, M. P. (1979). International research collaboration. Science, 9, 481-487.

Gauffriau, M., \& Larsen, P. O. (2005). Counting methods are decisive for rankings based on publication and citation studies. Scientometrics, 64(1), 85-93.

Gauffriau, M., Larsen, P. O., Maye, I., Roulin-Perriard, A., \& Von Ins, M. (2007). Publication, cooperation and productivity measures in scientific research. Scientometrics, 73, 175-214. doi:10.1007/s11192007-1800-2.

Gevers, M. (2014). Scientific performance indicators: A critical appraisal and a country-by-country analysis. In Bibliometrics: Use and abuse in the review of research performance (Vol. 37). Portland: WennerGren International Series.

Glänzel, W., \& Schubert, A. (2001). Double effort = double impact? A critical view at international coauthorship in chemistry. Scientometrics, 50(2), 185-202.

Glossary Incites. http://incites.isiknowledge.com/common/help/h_glossary.htmlAcess: http://www.portland press.com//pp/books/online/wg87/default.htm.

Katz, J. S., \& Martin, B. R. (1997). What is research collaboration? Research Policy, 26, 1-18.

King, D. A. (2004). The scientific impact of nations. Nature, 430, 311-316.

Larsen, P. O., \& Von Ins, M. (2010). The rate of growth in scientific publication and the decline in coverage provided by Science Citation Index. Scientometrics, 84, 575-603. doi:10.1007/s11192-010-0202-z.

Leite, L., Mugnaini, R., \& Leta, J. (2011). A new indicator for international visibility: Exploring Brazilian scientific community. Scientometrics, 88, 311-319.

Luukkonen, T., Persson, O., \& Silvertsen, G. (1992). Understanding patterns of international scientific collaboration. Science, Technology and Human Values, 17, 101-126.

Martin-Sempere, M. J., Rey-Rocha, J., \& Garzon-Garcia, B. (2002). The effect of team consolidation on research collaboration and performance of scientists. Case study of Spanish University researchers in Geology. Scientometrics, 55(3), 377-394.

May, R. M. (1997). The scientific wealth of nations. Science, 275, 793-796.

Meneghini, R. (2010). Publication in a Brazilian journal by Brazilian scientists whose papers have international impact. Brazilian Journal of Medical and Biological Research, 43, 812-815.

Meneghini, R., Packer, A. L., \& Nassi-Caló, L. (2008). Articles by Latin American authors in prestigious journals have fewer citations. PLoS One, 3(11), e3804.

Narin, K., Stevens, K., \& Whitlow, E. S. (1991). Scientific cooperation in Europe and the citation of multinationally authored papers. Scientometrics, 21, 313-323.

Persson, O., Glänzel, W., \& Danell, R. (2004). Inflationary bibliometric values: The role of scientific collaboration and the need for relative indicators in evaluative studies. Scientometrics, 60(3), 421-432.

Press, W. H. (2013). What's so special about science (and how much should we spend on it?). Science, 342, 817-822.

Richmond, G. (2016). Global science engagement. Science, 351(6272), 427.

Smith, M. J., Weinberger, C., Bruna, E. M., \& Allesina, S. (2014). The scientific impact of Nations: Journal placement and citation performance. PLoS One, 9, 1-6. 
Subramanyam, K. (1983). Bibliometrics studies of research collaboration: A review. Journal of Information Science, 19, 35-44.

Tahamtan, I., Afshar, A. S., \& Ahamdzadeh, K. (2016). Factors affecting number of citations: A comprehensive review of the literature. Scientometrics, 107, 1195-1225. doi:10.1007/s11192-016-1889-2.

UNESCO Science Report (2010). United Nations Educational, Scientific, and Cultural Organization. http:// www.unesco.org/new/en/natural-sciences/science-technology/prospective-studies/unesco-sciencereport/unesco-science-report-2010/.

Van Raan, A. (1997). Science as an international enterprise. Science Public Policy, 24, 290-300.

Van Raan, A. (1998). The influence of international collaboration on the impact of research results. Scientometrics, 42(3), 423-428.

Wadworth, J. (2014). 2014 Global R\&D funding forecast. R\&D Magazine. https://www.battelle.org/docs/ tpp/2014_global_rd_funding_forecast.pdf.

Wagner, C. S., Park, H. W., \& Leydesdorff, L. (2015). The continuing growth of global cooperation networks in research: A conundrum for national governments. PLoS One, doi:10.1371/journal.pone. 0131816.

World Bank (2015). World development indicators, 2015. http://data.worldbank.org/topic/science-andtechnology. 